\title{
O processo de trabalho de um time de medicação na perspectiva de uma pesquisa-ação
}

\section{The work process of a medication team from an action research perspective}

\author{
Viviane Saraiva de Almeidal', Marilda Andrade², Danielle Lemos Querido', Marialda Moreira Christoffel', \\ Angelina Maria Aparecida Alves ${ }^{1}$, Ana Paula Vieira dos Santos Esteves ${ }^{1}$
}

\section{RESUMO}

Objetivou-se descrever e analisar o processo de trabalho do time de medicaçáo na unidade de terapia intensiva neonatal. Pesquisa-ação realizada com 21 enfermeiros de uma unidade neonatal de um hospital universitário. Foram realizadas entrevistas, pesquisa documental, observação sistemática e seminários para a coleta e análise temática dos dados. O estudo apontou três categorias: dificuldades encontradas no processo de trabalho do time de medicação; caracterização dos recursos materiais e ambiente de trabalho; padronização do processo de trabalho do time de medicação. As principais barreiras apontadas foram a falta de padronização do processo de trabalho e a ausência e/ou atualização de instrumentos que subsidiam sua prática. Desta forma, a partir das açôes do grupo foram elaborados os seguintes produtos: fluxograma do processo de trabalho; construçáo e padronizaçáo de impressos; atualização de Protocolos Operacionais Padrão e atribuiçôes da equipe de enfermagem; padronização de material de almoxarifado, farmácia e apoio.

Descritores: Segurança do Paciente; Sistemas de Medicação; Terapia Intensiva Neonatal; Fluxo de trabalho; Enfermagem Neonatal.

\section{ABSTRACT}

This study aimed to describe and analyze the work process of the medication team in a neonatal intensive care unit. This action research was carried out with the participation of 21 nurses at a university hospital neonatal unit. Interviews were conducted, along with documental research, systematic observation and workshops for data collection and thematic analysis. The study indicated three categories: difficulties encountered in the work process of the medication team; description of material resources and working environment; and standardization of the work process of the medication team. The principal barriers indicated were lack of standardization of the work process and the absence and/or updating of instruments supporting work practices. As such, the following products were elaborated from the group actions: work process flowchart; development and standardization of printouts; updating of Standard Operational Protocols and duties of the nursing team; and standardization of storeroom, pharmacy and support material.

Descriptors: Patient Safety; Medication Systems; Intensive Care, Neonatal; Workflow; Neonatal Nursing.

\footnotetext{
${ }^{1}$ Universidade Federal do Rio de Janeiro - Rio de Janeiro (RJ), Brasil. E-mails: vivianesaraiva@hotmail.com, danyquerido@me.ufri.br, marialda.ufri@gmail.com, algelmalves@hotmail.com, anapaulaesteves@me.ufri.br

${ }^{2}$ Universidade Federal Fluminense - Niterói (RJ), Brasil. E-mail: marildaandrade@uol.com.br
}

Como citar este artigo: Almeida VS, Andrade M, Querido DL, Christoffel MM, Alves AMA, Esteves APVS. O processo de trabalho de um time de medicação na perspectiva de uma pesquisa-ação. Rev. Eletr. Enferm. [Internet]. 2019 [acesso em: ];21:54575. Disponível em: https://doi.org/10.5216/ree.v21.54575 


\section{INTRODUÇÃO}

Para promover a segurança no uso de medicamentos, deve-se buscar a compreensão dos fatores humanos no uso de tecnologias e controle das condiçóes ambientais. Apesar dos benefícios, este processo ainda é vulnerável e oferece riscos, podendo resultar em efeitos indesejados no cuidado ${ }^{(1)}$.

Dentre os riscos indesejados estão os Eventos Adversos Relacionados a Medicamentos (EARM), frequentes no mundo inteiro. Estudo revela que estes incidentes são os mais frequentes em Unidade de Terapia Intensiva Neonatal (UTIN), tendo como causa principal as atividades humanas na prestação do cuidado ${ }^{(1)}$, estimando-se que a probabilidade de erros com potencial para causar danos seja três vezes maior em crianças do que em adultos hospitalizados $^{(2)}$, tendo oito vezes mais chances de ocorrer em UTIN do que nos demais pacientes internados ${ }^{(3,4)}$.

Estudo realizado em uma UTIN revelou que os incidentes mais frequentes com medicamentos estão relacionados a dosagem incorreta ou inadequada (média de 38\%), seguidos da omissão, falha na técnica de administração e via de administração errada ${ }^{(1)}$. Os recém-nascidos (RN) prematuros são os mais suscetíveis devido a gravidade do quadro, o qual demanda tempo de internação prolongado sendo necessárias inúmeras intervençóes para seu tratamento e recuperação ${ }^{(3-5)}$. Além disso, as dosagens, específicas a este grupo de pacientes, influencia diretamente na necessidade de maior cuidado no preparo e administração de medicamentos ${ }^{(1)}$.

$\mathrm{Na}$ literatura internacional, o último Guideline (2011) preconiza que equipes de terapia intravenosa, denominadas IV Team, especializadas, se mostram eficazes na redução da incidência de infecçóes relacionadas a cateter, complicaçôes e custos associados a este procedimento. Além disso, o risco de infecção aumenta, dentre outros motivos, com a redução de pessoal de enfermagem especializado ${ }^{(6)}$.

Assim, visando a segurança do paciente e a redução de EARM na UTIN, foi criado o "time de medicação" (TM), constituído por enfermeiros dedicados exclusivamente ao preparo, discussão e revisão do sistema de medicação, como uma estratégia de reduzir danos a este grupo mais vulnerável.

A criação de um grupo especializado no tema é importante, pois a organização do processo de trabalho em todas as fases da terapia medicamentosa, varia de acordo com cada instituição, dificultando o estabelecimento de uma conduta padronizada, aumentando custos e possíveis riscos que comprometem a segurança do paciente ${ }^{(7)}$.

O TM atua como uma espécie de "consultor/orientador", baseado nas boas práticas e na literatura, analisando e tomando decisóes relativas a todo o processo de terapia medicamentosa ${ }^{(8)}$. O grupo está envolvido em todas as etapas do sistema de medicação da unidade, incluindo a prescrição, preparo, dispensação e administração de medicamentos.

A concepção de um time contribui de forma significativa na diminuição da variabilidade das práticas profissionais da enfermagem, proporcionando melhoria da qualidade da assistência prestada e reduzindo riscos e complicaçóes na administração de medicamentos, além de propiciar o desenvolvimento, educação, políticas e diretrizes relacionadas ao tema ${ }^{(9)}$.

Os processos de trabalho apresentam-se como uma tecnologia em saúde importante voltada para a segurança do paciente ${ }^{(10)}$. Entretanto, há uma lacuna na literatura que náo descreve o trabalho de times voltados para terapia medicamentosa. A busca nas bases de dados registra a ocorrência de outros times, porém nenhum deles ligado à temática ${ }^{(11,12)}$. Uma revisão integrativa recente indica a carência de estudos com fortes níveis de evidência no cenário brasileiro ${ }^{(13)}$.

Diante do exposto observou-se a necessidade de compreender melhor como se processa e quais as possíveis vulnerabilidades no processo de trabalho do time, estabelecendo-se o seguinte objetivo de estudo: descrever e analisar o processo de trabalho do time de medicação na UTIN.

\section{MÉTODO}

Pesquisa-ação (PA) descritiva de abordagem qualitativa. O cenário foi a UTIN do tipo II de um hospital universitário do estado do Rio de Janeiro, unidade com capacidade para 25 leitos, que atende em sua maioria $\mathrm{RN}$ prematuros ou a termo com instabilidade clínicas/fisiológicas.

$\mathrm{Na}$ PA é considerada a subjetividade dos sujeitos envolvidos, com o reconhecimento da necessidade real de mudança ${ }^{(14)}$, aspecto importante a ser considerado na estratégia de pesquisa, uma vez que na área organizacional há diferentes grupos de trabalho, onde as metas e decisóes não podem ser executadas sem a participação de todos os integrantes ${ }^{(15)}$. Recomenda-se a introdução de métodos participativos, com intuito de aumentar a produtividade e melhorias no trabalho ${ }^{(15)}$.

Este tipo de estudo considera no contexto organizacional a priorização na participação dos representantes com interesses no estudo, que trazem experiências, valores, comportamento e percepçóes peculiares. Seu princípio consiste na colaboração de pesquisadores e membros interessados; definição do problema; busca de soluçóes; e aprofundamento do conhecimento disponível, acompanhado de práticas pedagógicas. Além disso, tem por objetivo provocar mudanças organizacionais através de novas tecnologias, circulação da informação, aprendizagem coletiva, organização do trabalho em grupos com reunião de competências variadas ${ }^{(15)}$.

$\mathrm{Na}$ PA os participantes da pesquisa são intencionalmente escolhidos em função da relevância em determinado assunto $^{(15)}$, neste caso os enfermeiros do TM, atenderam os seguintes critérios de inclusão: especialização ou curso stricto sensu na área neonatal; experiência de, no mínimo, dois anos na terapia medicamentosa em neonatologia. Foram excluídos 
aqueles em licença médica ou ausentes por qualquer motivo durante o período da coleta de dados. Participaram do estudo 21 enfermeiros, que corresponde a todos os enfermeiros que compóem o TM e que atenderam os critérios pré-estabelecidos. A livre participação e o anonimato dos dados foram garantidos por meio da adoção de código alfanumérico composto pela letra $\mathrm{T}$ (Time) e um número arábico de acordo com a sequência de realizaçáo das entrevistas.

A coleta de dados ocorreu no ano de 2014 e será detalhada de acordo com as fases correspondentes no Quadro 1.

Com o agrupamento dos dados obtidos, os resultados foram organizados conforme as etapas de análise temática: pré-análise, exploração do material, tratamento dos resultados, inferência e interpretação, onde se buscou identificar e codificar os temas emergentes para, posteriormente, agrupá-los por similaridade e permitindo a elaboraçáo de três categorias que auxiliaram na descrição e compreensão do fenômeno investigado ${ }^{(16)}$, e que foram apresentadas por meio de dados descritivos, figuras e quadros nos resultados.

$\mathrm{O}$ estudo seguiu as recomendaçôes das Diretrizes e Normas da Pesquisa envolvendo Seres Humanos, apresentadas na Resolução no 466/2012 e foi aprovado pelo Comitê de Ética e Pesquisa da instituição, sob o parecer no 146.409 e no CAAE: 10209312500005259.

Quadro 1. Etapas da Pesquisa. Rio de Janeiro, RJ, Brasil, 2014.

\section{Fase}

Atividade

A coleta de dados nesta fase foi realizada no período de março a maio de 2014, por meio de entrevistas semiestruturadas composta por um formulário de perfil socioprofissional e questões relacionadas às barreiras, recursos e outros fatores que interferem no processo de trabalho do TM.

Fase

Exploratória

(Preliminar)

Neste mesmo período, em paralelo, procedeu-se ao levantamento e coleta de todos os documentos utilizados, criados ou que dizem respeito ao grupo: POP, instrumentos de comunicação, livros de ordens e ocorrências de enfermagem, atas de reunião do time e impressos utilizados na unidade. Além disso, foi realizada ainda, a observação sistemática pelo pesquisador principal, utilizando roteiro estruturado relacionado a sala de medicação; aos recursos materiais utilizados; e ao processo de trabalho do TM.

O roteiro baseou-se no sistema de medicação proposto pela Joint Commission on Accreditation of Healthcare Organizations (JCAHO): prescrição, preparo e dispensação, administração de medicamentos e monito-

Fase

Exploratória (Preliminar) ramento do paciente em relação aos efeitos dos mesmos.

Além disso, foi incorporado a esse roteiro um quinto processo denominado "transcrição de medicamentos", que ocorre no cenário do estudo, onde o profissional da equipe de enfermagem copia de forma sistematizada (por horário, por nome do paciente, por medicação) os dados referentes a medicação prescrita para um formulário exclusivo da equipe de enfermagem.

Após a fase exploratória preliminar, no período de junho a agosto de 2014 foi realizada uma adaptação dos seminários da pesquisa-ação à rotina de reuniões periódicas do time no serviço. Cinco encontros previamente agendados no cronograma da unidade, foram utilizados para esta fase exploratória ( $1^{\circ}$ e $2^{\circ}$ encontros) e para a fase ação ( $3^{\circ}, 4^{\circ}, 5^{\circ}$ encontros).

Os encontros também subsidiaram outras fases da pesquisa-ação (coleta de dados, aprendizagem, ação e avaliação).

Fase $\quad$ Cada encontro durou em média duas horas e foram gravados em áudio, com posterior transcrição na íntegra, exploratória além de registrados em diário de campo. Todos foram realizados em sala de reuniões anexa a UTIN, sem (Encontros)

loe $2^{\circ}$ encontros Seminários interferências externas.

Dando início a fase exploratória (encontros), nos dois primeiros encontros foram discutidos os seguintes aspectos: objetivos do estudo; interesses do grupo; regras da participação e definição da operacionalização dos encontros (data, local, horário e duração); levantamento das necessidades e problemas; listadas tarefas e atribuições do pesquisador principal e dos enfermeiros do time dentro da pesquisa.

As discussões foram norteadas por perguntas disparadoras, construídas de acordo com os resultados da análise dos dados coletados nas entrevistas, pesquisa documental e observação.

Nestes grupos os enfermeiros do time puderam refletir sobre a prática comum a fim de identificar pontos vulneráveis e planejar ações relacionadas aos temas elencados pelo grupo como os mais relevantes.

A colocação do problema foi determinada pela falta de padronização e a necessidade de atualização dos participantes sobre temas importantes relacionados ao seu processo de trabalho. 
Quadro l. Continuação.

\begin{tabular}{|c|c|}
\hline Fase & Atividade \\
\hline $\begin{array}{l}\text { Fase Ação } \\
30,4^{\circ} \text { e } 5^{\circ} \\
\text { encontros } \\
\text { Seminários }\end{array}$ & $\begin{array}{l}\text { A partir dos dados obtidos na fase exploratória (preliminar e encontros) os temas prioritários estabelecidos } \\
\text { para a fase de ação foram: construção de impressos (POP relacionados a medicamentos e outros impressos } \\
\text { específicos que dão suporte ao trabalho do grupo); atualização de outros POP e atribuições da equipe de } \\
\text { enfermagem; padronização de impressos, material de almoxarifado e de farmácia e de apoio já adotados } \\
\text { pela instituição. } \\
\text { Para tal, os enfermeiros se dividiram por turno de trabalho e afinidade para confeccionar ou atualizar os } \\
\text { materiais. } \\
\text { A troca de experiências entre os participantes propiciou rico momento de aprendizagem, que foi subsidiado } \\
\text { também por uma atualização teórica quanto ao uso de medicamentos na UTIN. No 5o encontro, o grupo } \\
\text { elaborou uma proposta para padronização do processo de trabalho do TM, por meio da construção de fluxo- } \\
\text { grama, com a descrição dos passos desde a fase da confecção da prescrição médica até o acompanhamento } \\
\text { de efeitos adversos, com base no material construído pelo grupo. }\end{array}$ \\
\hline $\begin{array}{l}\text { Fase } \\
\text { Avaliação } \\
\text { 6ํencontro }\end{array}$ & $\begin{array}{l}\text { Todos os materiais construídos pelo grupo foram apresentados e avaliados por outros enfermeiros da uni- } \\
\text { dade que não integram o TM. Esses instrumentos foram colocados em prática com intuito de funcionar } \\
\text { como orientador, permitindo que qualquer profissional siga um roteiro estruturado (antes inexistente), } \\
\text { visando reduzir os riscos de erros durante o processo de trabalho do time. }\end{array}$ \\
\hline
\end{tabular}

\section{RESULTADOS}

A partir da aplicação do formulário de caracterização do perfil socioprofissional dos 21 participantes, observou-se que estes encontram-se em uma faixa etária de 30 a 60 anos, com média de 42 anos. $\mathrm{O}$ maior tempo de formação profissional foi de 30 anos e o menor de um ano, com média de 12 anos. O tempo de atuação na instituição e na área de terapia intensiva neonatal variou de dois a 25 anos, com média de seis anos na UTIN. O tempo de atuação no TM foi em média dois anos, onde 15 profissionais referem participação em eventos de classe para atualizaçóes, 12 possuem cursos de aperfeiçoamento ou atualização na área, 14 possuem qualificação lato sensu e sete stricto sensu.

Os dados gerados resultaram em três categorias descritas a seguir.

\section{As dificuldades encontradas no processo de trabalho do time de medicação}

Os participantes relataram como principais dificuldades enfrentadas: a falta de padronização do trabalho do grupo, tanto no que tange à assistência propriamente dita quanto à padronização de impressos, recursos materiais utilizados pelo grupo, excesso de ruídos externos durante o preparo de medicamentos na sala de medicação:

\section{[...] isso [falta de padronização] dificulta muito o trabalho do time porque se tivesse escrito tinha uma regra (T3).}

[...] tem muita coisa que náo tá escrita, que a gente faz como acha que é [...] inclusive com os impressos que quando tem gente nova ou residente e nâo tem uma lista [...] (T4).
[...] não tem escrito os materiais que a gente deve usar pra preparar, tipo a seringa que vai administrar no PICC tem que ser de 10 ou 20 [ml], a gente sabe porque tá acostumado (T1).

[...] uma coisa que atrapalha é o barulho na hora de preparar medicação, tira atenção da gente (T20).

\section{Caracterização dos recursos materiais e ambiente de trabalho do time de medicação}

Nas falas obtidas dos participantes, foi destacada a importância de traçar estratégias para minimizar as dificuldades eleitas como prioritárias, entre elas estava a confecção dos Quadros 2 e 3 que caracterizam os recursos e ambiente de trabalho do time de medicação.

\section{Padronização do processo de trabalho do time de medicação}

A Figura 1 apresenta um fluxograma descrevendo a sequência operacional das etapas do sistema de medicação na unidade, a interação dos profissionais entre si e com os enfermeiros do time.

Para tornar mais claro as funçóes de cada membro da equipe de enfermagem no sistema de medicação, na UTIN, foram atualizadas suas atribuiçôes, ficando assim definidas no Quadro 4.

A partir desta organização foi possível descrever também as atribuiçóes de outros profissionais que atuam no sistema de medicação, que tem início no momento em que o médico realiza prescrição no sistema on-line.

O Serviço de Farmácia é responsável por receber as prescriçôes, imediamente após finalização pelo médico. 
Quadro 2. Caracterização estrutural e funcional da Sala de Medicação e a descrição, segundo a perspectiva dos participantes. Rio de Janeiro, RJ, Brasil, 2014.

\begin{tabular}{|c|c|}
\hline Fala dos Participantes & Caracterização da Sala de medicação \\
\hline \multirow[t]{2}{*}{$\begin{array}{l}\text { Na sala a gente só } \\
\text { prepara medicação, é } \\
\text { só pra isso. Mas tem } \\
\text { medicação que é no } \\
\text { leito, pra nebulização, } \\
\text { pomada (Tl). }\end{array}$} & $\begin{array}{l}\text { Finalidade } \\
\text { - Exclusiva para o preparo de soluções parenterais e soluções enterais de RN internados em UTIN. } \\
\text { - Medicamentos tópicos e inalatórios são manipulados à beira do leito do RN e os materiais uti- } \\
\text { lizados para sua administração são de uso individual, sendo este procedimento, uma atribuição } \\
\text { de técnico em enfermagem. }\end{array}$ \\
\hline & $\begin{array}{l}\text { Medida } \\
-5,3 \mathrm{~m}^{2}\end{array}$ \\
\hline $\begin{array}{l}\text { [...] a luz da sala é boa, } \\
\text { não tem problema não, } \\
\text { e o ar funciona direito, } \\
\text { até é bem geladinho, } \\
\text { tem que anotar a } \\
\text { temperatura da sala no } \\
\text { SD e SN [...] (T8). }\end{array}$ & $\begin{array}{l}\text { Iluminação, Ventilação e Climatização } \\
\text { - A iluminação da sala se dá por lâmpadas fluorescentes, adequadas e em números suficientes. } \\
\text { - Não há janelas, apenas um pequeno basculante na porta para que o enfermeiro da medicação } \\
\text { possa se comunicar com o interior da UTIN, em caso de urgência. } \\
\text { - A ventilação ocorre por abertura na parte superior da divisória que delimita a área de preparo e } \\
\text { que permite a comunicação com a área de internação da UTIN. } \\
\text { - A climatização se dá através de saída do ar condicionado central, instalada no teto da sala de } \\
\text { preparo de medicação. }\end{array}$ \\
\hline $\begin{array}{l}\text { [...] a gente guarda na } \\
\text { estante como se fosse } \\
\text { uma reservinha de } \\
\text { medicamentos [...] (T8). }\end{array}$ & $\begin{array}{l}\text { Mobiliário e Recursos } \\
\text { - A sala dispõe de pia e recipientes para sabão e papel toalha, afixados na parede. Conta ainda } \\
\text { com estantes para armazenamento de medicamentos via parenteral e enteral, e recipiente para } \\
\text { descarte de material perfurocortante, além de divisória de inox na pia, separando-a da bancada } \\
\text { seca, destinada ao preparo dos medicamentos. }\end{array}$ \\
\hline
\end{tabular}

Quadro 3. Caracterização dos recursos materiais e a descrição segundo a perspectiva dos participantes. Rio de Janeiro, RJ, Brasil, 2014.

\section{Falas dos Participantes}

[...] é papel que não acaba mais, tudo é papel, mas serve também pra nosso controle, porque temos muitos pra tirar dúvida e consultar e outros pra preencher que a farmácia e almoxarifado exigem [...] (T2).

\section{Recursos Materiais utilizados pelo Time de Medicação}

Mapa de medicação; quadro para administração e conservação de medicamentos de uso oral; mapa de solicitação de medicamentos manipulados a farmácia; quadro de medicamentos usualmente manipulados na unidade neonatal; controle de temperatura do refrigerador; quadro de equipamentos padronizados para uso na medicação; quadro de materiais padronizados para o uso na medicação; quadro de diluição de medicamentos (nome do medicamento), armazenamento, diluição, soluções compatíveis, tempo de infusão, estabilidade após reconstituição, observações e incompatibilidade).

POP de preparo de medicamentos via oral (VO), intramuscular (IM), endovenosa (EV), subcutânea (SC), e sublingual (SL); formulário de notificação de EARM com medicamentos; POP de monitoramento do paciente quanto a efeitos adversos com medicamento.

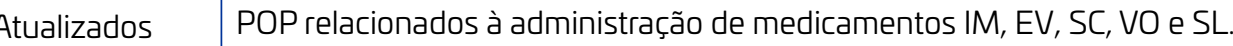

\begin{tabular}{l|l}
\cline { 2 - 2 } pelo time & Atribuições da equipe de enfermagem.
\end{tabular}

Padronizados $\quad$ Pedidos de psicotrópicos e medicamentos especiais; prescrição médica; pela instituição balanço hídrico. 
Quadro 3. Continuação.

\section{Falas dos Participantes}

$[\ldots .$.$] a gente usa pra$ preparar seringa, agulha, álcool, gaze, luva estéril, bandeja, equipo de soro (T2l).

A medicação a gente coloca em cubase as HVs [hidratação venosa] nas bandejas $[\ldots](\mathrm{TIO})$.

\section{Recursos Materiais utilizados pelo Time de Medicação}

Seringas, agulha, extensores, cânulas, equipos para bomba infusora, gorros, máscara, luvas estéreis, luvas de procedimento, gaze estéril, gaze não estéril, papel toalha, fita adesiva, dispositivo, dispositivo para irrigação em frascos de solução em sistema fechado, e escovas de degermação.

\begin{tabular}{l|l} 
Material de & estéril, papel toalha, fita adesiva, dispositivo, dispositivo para irrigação em
\end{tabular}

Material de

Soluções antissépticas utilizadas durante o preparo de medicamentos: álcool a $70 \%$ e clorexidina 0,5\% solicitados semanalmente ao Serviço de Farmácia.

Material de Apoio

Cubas, bandejas, cálices graduados, potes e sacos de material plástico.

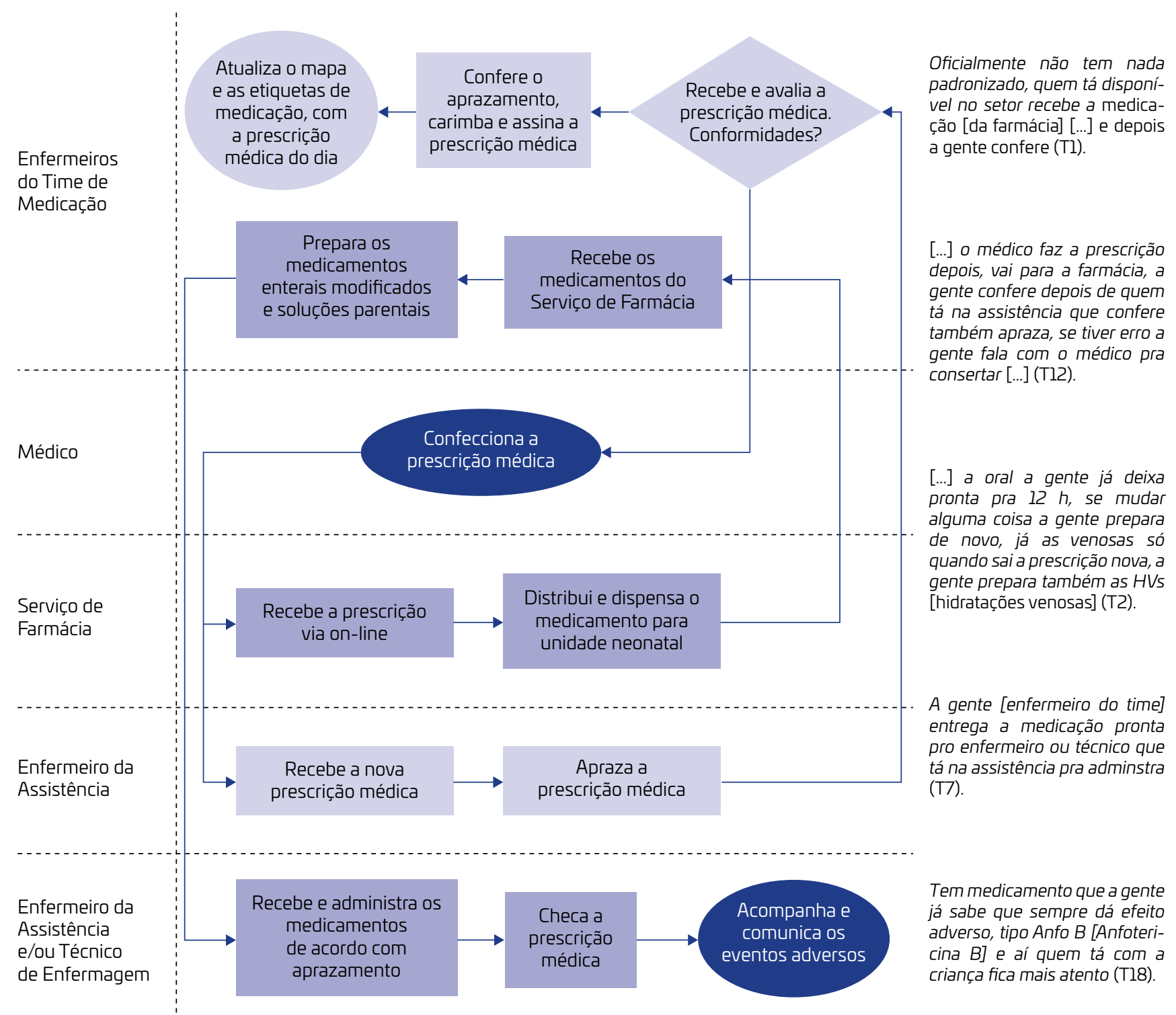

Figura 1. Fluxograma do processo de trabalho do time de medicação da UTIN e a descrição segundo a perspectiva dos participantes correspondentes. Rio de Janeiro, RJ, Brasil, 2014. 
Paralelo a isto, as prescriçóes são impressas e entregues ao enfermeiro da assistência, que realiza a primeira checagem. Caso esteja correta, este realiza o aprazamento, considerando, dentre outros, o intervalo de doses prescrito e possíveis interações com alimentação ou outros medicamentos, carimba e assina.

O enfermeiro da medicação realiza nova conferência, desta forma sendo "dupla" a checagem, carimba e assina. Caso algum item não esteja em conformidade, a prescrição médica retorna para a fase "confecciona a prescrição médica". Caso ocorra alteração, esta não é feita no sistema, sendo o medicamento suspenso, alterado ou acrescido de forma manuscrita na prescrição. Em seguida, o mapa de medicação é novamente conferido e são efetuadas as correçóes e atualizaçóes pelo enfermeiro da medicação.

A fase de transcrição, foi consenso no grupo, como uma etapa importante dentro do sistema de medicação, uma vez que, a indisponibilidade frequente da prescrição para o preparo dos medicamentos, devido a ajustes pela equipe médica, inviabiliza a execução da mesma.

Neste estudo, o papel da farmácia é restrito, limitando-se prioritariamente na dispensação de medicamentos, com dificuldade em realizar revisão das prescriçôes. $\mathrm{O}$ serviço de farmácia em posse da prescriçáo recebida on-line, seleciona os medicamentos para uso nas 24 horas e os dispensa de forma individualizada, com a cópia da prescrição. Entretanto, observa-se que, algumas vezes, a quantidade de medicamentos dispensada é aquém da necessidade, devido a falta de previsão da quantidade utilizada para preenchimento de equipos e perfusores.

Quadro 4. Atribuições da Equipe de Enfermagem. Rio de Janeiro, RJ, Brasil, 2014.

\begin{tabular}{|c|c|}
\hline Função & Atividade \\
\hline $\begin{array}{l}\text { Enfermeiros da } \\
\text { medicação }\end{array}$ & $\begin{array}{l}\text { Realizam o preparo de medicamen- } \\
\text { tos na sala de medicação e conta com } \\
24 \text { profissionais. }\end{array}$ \\
\hline $\begin{array}{l}\text { eiros da } \\
\text { ncia }\end{array}$ & $\begin{array}{l}\text { Prestam assistência/atuam no cuidado } \\
\text { direto aos RN mais graves ou executam } \\
\text { procedimentos de maior complexidade; } \\
\text { além de administrar medicamentos em } \\
\text { cateteres venosos profundos, contan- } \\
\text { do com } 27 \text { profissionais. }\end{array}$ \\
\hline $\begin{array}{l}\text { Técnicos de } \\
\text { enfermagem }\end{array}$ & $\begin{array}{l}\text { Prestam assistência/atuam no cuidado } \\
\text { direto de RN de baixa e média complex- } \\
\text { idades e administram medicamentos } \\
\text { VO, SC, IM, SL, tópicos, inalatórios e EV } \\
\text { em cateteres venosos periféricos, equi- } \\
\text { pe de } 36 \text { profissionais. }\end{array}$ \\
\hline
\end{tabular}

O enfermeiro da medicaçáo no período diurno recebe os medicamentos e as cópias das prescriçōes, enviadas pelo Serviço de Farmácia, e realiza nova conferência destes, observando a identificação do RN, nome do medicamento, forma farmacêutica prescrita, dose, intervalo das doses, prazo de validade, temperatura e embalagem.

Como estratégia preliminar ao preparo de medicamentos, em virtude dos ruídos, foi estabelecida, como estratégia de melhoria, a confecçáo de uma placa com o aviso "Em preparo de medicamentos. Não entre". Além disso, foi adotado como rotina o trancamento da sala de medicaçáo durante o preparo, também com o objetivo de minimizar os ruídos advindos do exterior.

Dando início aos procedimentos preliminares ao preparo de medicamentos são confeccionados os rótulos e etiquetas de identificação dos medicamentos, tendo o grupo optado por acrescentar, na etiqueta, o nome do profissional que realizou o preparo do medicamento.

Os enfermeiros do TM realizam o preparo dos medicamentos enterais após a confecção da prescrição, utilizando-se técnica estéril ficando os mesmos armazenados no refrigerador por até 12 horas, sendo distribuídos de acordo com o aprazamento. Já as soluçôes parenterais de infusão contínua ou administraçáo intermitente sáo preparadas conforme horário da prescrição.

O enfermeiro da assistência ou o técnico em enfermagem, escalado no cuidado direto ao $\mathrm{RN}$, recebe o medicamento em cubas individuais, protegidas por plástico, com a orientação de que o medicamento seja logo administrado e não fique sobre a bancada, a fim de manter sua integridade. Após receberem o medicamento, realizam a conferência com a prescrição e $\mathrm{o}(\mathrm{s})$ administram. O técnico em enfermagem está habilitado a administrar medicamentos em todas as vias, exceto em cateteres venosos profundos (CVP).

Após administrar o medicamento, o profissional checa a prescrição médica, e o enfermeiro da medicação verifica o registro do mesmo no balanço hídrico e na prescrição. Após realizada a administração, os profissionais acompanham atentamente a evoluçáo do RN, e comunicam EARM, caso haja. Entretanto, esta comunicação é frequentemente realizada de forma verbal e, ocasionalmente, no prontuário. Isto ocorre mesmo quando é necessária a prescrição de medicamentos em decorrência do EARM, náo sendo possível relacioná-lo, posteriormente, ao evento ocorrido.

Tendo em vista a importância do monitoramento sistemático e a constatação da sua não realização nesta fase do sistema de medicação, o grupo deliberou por criar um formulário de notificação, que contemplasse os EARM e um POP de monitoramento do paciente.

Ressalta-se ainda que o estudo evidenciou a importância na redução da rotatividade de enfermeiros a cada turno, para o preparo de medicamentos. Isto permite que o profissional 
adquira conhecimentos, expertise e habilidades com relação aos medicamentos mais usuais no setor, incluindo a análise e transcrição da prescrição médica.

\section{DISCUSSÃO}

O sistema de medicação de uma UTIN deve se pautar no trabalho em equipe, que transcende a etapa de prescrição e administração, incluindo um processo de trabalho contínuo, que envolve a construção de protocolos clínicos de trabalho, impressos específicos, discussóes e sensibilização de toda a equipe envolvida neste processo. A padronizaçáo promove a melhoria da qualidade e do andamento das atividades relacionadas, através de guias ou fluxos para os procedimentos desenvolvidos, proporcionando agilidade e eficiência ${ }^{(8)}$.

Dentre os procedimentos atribuídos ao time de medicação está a avaliação de recursos materiais utilizados no processo dentro da unidade ${ }^{(8)}$. Além dos recursos utilizados, outro ponto importante é o ambiente onde o grupo desenvolve suas atividades, a sala de medicação.

A sala de serviços de enfermagem destinada para o armazenamento e preparo de medicação, na área de neonatologia, deve ter uma dimensão mínima de $6,0 \mathrm{~m}^{2(17)}$. Mesmo com ampliação realizada recentemente, o espaço ainda não está adequado à legislação, verificando-se ainda disposição inadequada do mobiliário (estantes, refrigerador e bancos) e dificuldade na circulação dos enfermeiros.

A área de preparo de soluçóes parenterais deve ser exclusiva para esta finalidade e o acesso restrito. A iluminação e ventilação devem ser suficientes para que a temperatura e a umidade do ar não deteriorem os medicamentos, e também possibilitem o desenvolvimento das atividades ${ }^{(4,18)}$.

Os ambientes necessitam de lavatórios/pias em número suficiente, com provisão de sabão, antisséptico e material para secagem das mãos. As soluçóes parenterais poderão ser armazenadas diretamente sobre estrados ou estantes. Os produtos para pronto uso podem ser armazenados em armário específico, dentro da sala de preparo $^{(18,19)}$.

A infraestrutura da sala de medicação é adequada quanto aos lavatórios, saboneteiras, estantes para armazenamento de medicamentos, recipiente para descarte de material perfuro cortante $^{(18)}$.

Durante a coleta de dados do estudo foi observada a presença de ruídos na unidade, que dificultam a concentração no preparo de medicamentos. Tal situação deve ser minimizada, e a equipe deve ser encorajada a eliminá-los para que o processo ocorra com segurança e qualidade, diminuindo os riscos para o paciente ${ }^{(20)}$.

A fase de transcrição dos medicamentos é prática comum da equipe de enfermagem, entretanto, esta ação pode causar danos importantes, dentre outros se destaca a possibilidade de troca do medicamento prescrito ou via de administração ${ }^{(21)}$.
Nesta transcrição, os rótulos confeccionados para as soluçóes parenterais de pequeno volume devem conter: nome completo do paciente, leito, nome do medicamento, dosagem, horário, via de administração e identificação do profissional que as preparou ${ }^{(18,20)}$.

$\mathrm{Na}$ instituição estudada a prescrição médica é eletrônica, tecnologia que pode contribuir para a prevenção de erros de medicaçáo derivados da escrita ou leitura da mesma na forma manuscrita ${ }^{(22)}$. Além disso, a denominação dos medicamentos padronizados pelo sistema eletrônico facilita sua identificação e contribui para a diminuição das chances de erros relacionados ao nome do medicamento.

Recomenda-se que toda alteração na prescrição seja realizada no sistema eletronicamente ${ }^{(23)}$, e imediatamente comunicada à equipe de enfermagem.

É recomendado também a dupla checagem principalmente em pacientes de risco, como os pacientes pediátricos ${ }^{(5,23)}$, sendo esta realizada sempre por dois enfermeiros antes de sua administração.

No momento da checagem, também é importante esclarecer as possíveis dúvidas sobre a indicação e posologia do medicamento antes da administração. Isto poderá auxiliar na não ocorrência, na fase de aprazamento, das interaçóes medicamentosas indesejáveis ${ }^{(19,23)}$.

Quanto ao papel do serviço de farmácia, recomenda-se que o farmacêutico observe a concentraçáo, viabilidade, compatibilidade físico-química e farmacológica dos componentes, dosagem, forma farmacêutica, via e horário de administração ${ }^{(21,24)}$. O sistema de distribuição de medicamentos individualizados, caracterizado pela distribuiçáo dos medicamentos prescritos por paciente, para cobertura das 24 horas, apresenta-se como mais seguro que o sistema de distribuição coletiva, porém, menos que o sistema de dose unitária ${ }^{(5,23)}$, pois neste último a dose do medicamento é dispensada pronta para ser administrada, sem necessidade de transferências, cálculos e manipulação prévia por parte da enfermagem antes da administração ${ }^{(23)}$.

No que se refere às fases de preparo e administração de medicamentos, estas constituem etapas potenciais para erros de medicação. O momento da administração é a última oportunidade de verificação e interrupção de possíveis erros, fundamental para redução de danos evitáveis ${ }^{(25)}$.

Nesse sentido, preparar o medicamento imediatamente antes da administração contribui sobremaneira para a não ocorrência do erro ${ }^{(19,23)}$. O transporte do medicamento da sala de preparo até o paciente deve ser realizado com os devidos cuidados para manter sua integridade físico-química e microbiológica ${ }^{(18)}$, devendo sempre que possível, ser levado direto para a incubadora do $\mathrm{RN}^{(20)}$.

A administração de medicamentos pode ser realizada legalmente pelo técnico em enfermagem, entretanto o enfermeiro deve supervisionar esta etapa, certificando-se 
do registro e da administraçáo, evitando a duplicação da administração do medicamento por outro profissional ${ }^{(19,23)}$. Além disso, é papel do enfermeiro capacitar e atualizar sua equipe em relaçấo ao processo de medicação.

A falta de monitoramento adequado de EARM revela a frágil conscientização dos profissionais envolvidos sobre a importância de tal ação para a segurança dos pacientes, para a qualidade da assistência prestada e para a eficácia do tratamento, considerando que muitas vezes essa etapa, acaba sendo realizada quando os efeitos indesejáveis aparecem, de forma evidente, após sua administraçấo ${ }^{(20)}$.

A falta de sistematização no monitoramento e comunicação dos EARM na UTIN dificulta a investigação e implementação de medidas que possam contribuir na prevenção de novos eventos, constataçáo baseada nos resultados coletados do monitoramento dos tipos e causas destas ocorrências na unidade.

Todo evento adverso envolvendo a terapia infusional deve ser devidamente investigado com base em registros do problema $^{(1,18)}$.

É importante destacar, neste estudo, o papel fundamental da equipe de enfermagem no sistema de medicação da UTIN, e a importância das interaçóes entre as diversas categorias profissionais, essenciais para terapia medicamentosa exitosa.

Dentro deste contexto, cabe ressaltar que a metodologia permitiu aos enfermeiros do time atuarem como agentes transformadores da sua própria prática, em um contexto organizacional específico.

\section{CONCLUSÃO}

Neste estudo foi possível reconhecer e reproduzir reflexôes sobre o processo de trabalho do TM. Dentro deste contexto, as principais dificuldades relatadas pelo grupo foram a falta de padronização do seu processo de trabalho, ausência e/ou atualização de vários instrumentos que subsidiam sua prática.

Assim, a partir da priorizaçáo de pontos vulneráveis específicos, foi elaborado um fluxograma, onde o contexto e a relaçáo com outros profissionais foi descrito a partir de esquema gráfico pautado nas fases do sistema de medicação, demostrando algumas falhas, principalmente no monitoramento dos eventos adversos e na revisão de prescriçóes pelo Serviço de Farmácia.

O método também possibilitou a implementação de açóes, correção de falhas e tomada de decisóes, geradas pelos próprios profissionais a partir de sua realidade, tornando o processo seguro e com qualidade, com impacto na atuaçáo e aprendizagem a partir de um plano de ação construído coletivamente, no qual os enfermeiros demonstraram conhecimento dos problemas relacionados ao seu cotidiano com a proposiçấo de estratégias exequíveis de melhorias tais como; a construção de impressos; atualização de POP e atribuiçóes da equipe de enfermagem; padronização de impressos, material de almoxarifado e de farmácia e de apoio já adotados pela instituição.

Uma das limitaçóes do estudo está relacionada ao pouco tempo disponível para a realização dos grupos, não permitindo uma análise mais profunda para os participantes. Contudo, trata-se de um estudo inédito sobre um time desta natureza, comprometido e incluído em todas as fases do sistema da medicação de uma unidade específica, cuja padronização das etapas de trabalho, construída pelos próprios sujeitos, fortalece o processo dentro da unidade, com vistas à redução de EARM.

Além disso, a divulgaçáo de uma nova tecnologia, criada a partir da formação de um time dedicado à terapia medicamentosa e a sistematizaçáo do processo de trabalho, agrega conhecimento na área de medicamentos, e fornece subsídios para a replicaçáo em outros grupos da mesma natureza.

\section{REFERÊNCIAS}

1. Lanzillotti LS, Seta MH, Andrade CLT, Mendes Junior WV. Adverse events and other incidents in neonatal intensive care units. Ciênc Saúde Colet [Internet]. 2015 [acesso em: 1 jul. 2018];20(3):937-46. Disponível em: http://www. scielo.br/scielo.php?script $=$ sci arttext $\&$ pid $=S 1413$ 812320150003009378lng=en. $\quad \underline{\text { http://dx.doi. }}$ org/10.1590/1413-81232015203.16912013.

2. Belela ASC, Pedreira MLG, Peterlini MAS. Erros de medicação em pediatria. Rev Bras Enferm [Internet]. 2011 [acesso em: 9 dez. 2018];64(3):563-569. Disponível em: http://www. scielo.br/scielo.php?script $=$ sci arttext\&pid $=$ S003471672011000300022\&lng=en. $\quad \underline{\text { http://dx.doi. }}$ org/10.1590/S0034-71672011000300022.

3. Ruiz MTE, Suñol MGM, Miguélez LMR, Ortiz ES, Urroz MI, Camino ML, et al. Los errores de tratamiento en una unidad neonatal, uno de los principales acontecimientos adversos. An Pediatr [Internet]. 2016 [acesso em: 9 dez. 2018];84(4):211-7. Disponível em: http://www.analesdepediatria. org/es-linkresolver-los-errores-tratamiento-unaunidad-S1695403315003707. http://dx.doi. org/10.1016/j.anpedi.2015.09.009.

4. Campino A, Arranz C, Unceta M, Sordo B, Pascual P, Lopez-de-Heredia I, et al. Medicine preparation errors in ten Spanish neonatal intensive care units. Eur J Pediatr [Internet]. 2016 [acesso em: 9 dez. 2018];175:203-10. Disponível em: https://www. ncbi.nlm.nih.gov/pubmed/26311566. http://dx.doi. org/10.1007/s00431-015-2615-4. 
5. Jain S, Guglani V. Patient safety in pediatrics and neonatal medication. J Med Research [Internet]. 2016 [acesso em: 9 dez. 2018];2(1):16-9. Disponível em: http://www. medicinearticle.com/JMR 201621 05.pdf.

6. O'Grady NP, Alexander M, Burns LA, Dellinger EP, Garland J, Heard SO, et al. Guidelines for the prevention of intravascular catheter-related infections. Clin Infect Dis [Internet]. 2011 [acesso em: 9 dez. 2018];52(9):162-93. Disponível em: https:// www.ncbi.nlm.nih.gov/pmc/articles/PMC3106269/. http://dx.doi.org/10.1093/cid/cir257.

7. Magalhães AMM, Moura GMSS, Pasin SS, Funcke LB, Pardal BM, Kreling A. The medication process, workload and patient safety in inpatient units. Rev Esc Enferm USP (Online) [Internet]. 2015 [acesso em: 8 jul. 2018];49(n.spe):43-50. Disponível em: scielo.br/scielo.php?script $=$ sci arttext \&pid $=S 0080$ 62342015000700043\&lng=en. $\quad$ http://dx.doi. org/10.1590/S0080-623420150000700007.

8. Almeida VS, Andrade M, Silva GRG, Monteiro JLS, Silva GD, Querido DL. The work process of a medication team at the neonatal intensive care unit. Rev. Enferm UFPE on line. [Internet]. 2013 [acesso em: 10 jul. 2018];(5):4299-303. Disponível em: http://www.scielo.br/scielo. php?pid $=$ S0080-62342011000200011\&script $=$ sci arttext\&tlng=en. $\quad$ http://dx.doi.org/10.1590/S008062342011000200011.

9. Wallis MC, McGrail M, Webster J, Marsh N, Gowardman J, Playford G, et al. Risk factors for peripheral intravenous catheter failure: a multivariate analysis of data from a randomized controlled trial. Infect Control Hosp Epidemiol [Internet]. 2014 [acesso em: 10 jul. 2018];35(1):63-8. Disponível em: https:// www.ncbi.nlm.nih.gov/pubmed/24334800. http:// dx.doi.org/10.1086/674398.

10. Resoluçáo de Diretoria Colegiada (RDC) no 36, de 25 de julho de 2013. (BR) [Internet]. Institui açôes para a segurança do paciente em serviços de saúde e dá outras providências. Diário Oficial da União. 26 jul. 2013 [acesso em: 8 jul. 2018]. Disponível em: http:// portal.anvisa.gov.br/documents/10181/2871504/ RDC 362013 COMP.pdf/36d809a4-e5ed-4835a375-3b3e93d74d5e.

11. Taguti PS, Dotti AZ, Araujo KP, Pariz PS, Dias GF, Kauss IAM, et al. The performance of a rapid response team in the management of code yellow events at a university hospital. Rev Bras Ter Intensive [Internet]. 2013 [acesso em: 11 jul. 2018];25(2):99-105. Disponível em: $\quad$ http://www.scielo.br/scielo.php?script=sci arttext\&pid=S0103-507X2013000200007\&lng=en. http://dx.doi.org/10.5935/0103-507X.20130020.
12. Carrier M, Fergusson DA, Huebsch LB, Mcdiarmid $S$, Scrivens N, Sabri E, et al. Outcomes in a nurseled peripherally inserted central catheter program: a retrospective cohort study. CMAJ open [Internet]. 2016 [acesso em: 11 jul. 2018];5(3):E535-39. Disponível em: https://www.ncbi.nlm.nih.gov/pubmed/28676535. http://dx.doi.org/10.9778/cmajo.20170010.

13. Gomes ATL, Assis YMS, Silva MF, Costa IKF, Feijão AR, Santos VEP. Medication Administration Erros: evidences and implications for patient safety. Cogitare Enferm [Internet]. 2016 [acesso em: 9 dez. 2018];21(3):1-11. Disponível em: https://revistas. ufpr.br/cogitare/article/view/44472. http://dx.doi. org/10.5380/ce.v21i3.44472.

14. Rocha BS, Munari DB, Ribeiro LCM, Rego PG. Evidence in nursing leadership development through action research: an integrative review. Rev Eletr Enf [Internet]. 2017 [acesso em: 9 dez. 2018];19:a41. Disponível em: https://www. revistas.ufg.br/fen/article/view/46827/24671. http:// dx.doi.org/10.5216/ree.v19.46827.

15. Thiollent, M. Metodologia da pesquisa-ação. $18^{a}$ ed. São Paulo: Cortez; 2011.

16. Minayo, MC. Pesquisa social: teoria, método e criatividade. Petrópolis: Vozes; 2016.

17. Resolução de Diretoria Colegiada (RDC) no 50, de 21 de fevereiro de 2002 (BR) [Internet]. Dispõe sobre o regulamento técnico para planejamento, programação, elaboração e avaliação de projetos físicos de estabelecimentos assistenciais de saúde. Diário Oficial da União. 21 fev. 2002 [acesso em: 10 jul. 2018]. Disponível em: $\quad$ http://bvsms.saude.gov.br/bvs/saudelegis/ anvisa/2002/anexo/anexo prt0050 2102 2002.pdf.

18. Resolução de Diretoria Colegiada no 45 , de 12 de março de 2003 (BR) [Internet]. Dispóe sobre o regulamento técnico de boas práticas de utilização das soluçôes parenterais e serviços de saúde. Diário Oficial da União. 12 mar. 2003 [acesso em: 20 jun. 2018]. Disponível em: https://www20.anvisa.gov.br/segurancadopaciente/ index.php/legislacao/item/resolucao-rdc-n-45-de-12de-marco-de-2003.

19. Galiza DDF, Moura OF, Barros VL, Luz GOA. Preparo e administração de medicamentos: erros cometidos pela equipe de enfermagem. Rev Bras Farm Hosp Serv Saúde [Internet]. 2014 [acesso em: 11 jul. 2018];5(2):45-50. Disponível em: http://www.sbrafh.org.br/v1/public/ artigos/2014050205000528BR.pdf.

20. Gaiva MA, Souza JS. Medication administration errors in neonatal intensive care units. Cienc Cuid Saúde [Internet]. 2015 [acesso em: 9 dez. 2018];14(3):1330-8. Disponívelem: http://periodicos.uem.br/ojs/index.php/ CiencCuidSaude/article/view/25445/pdf 377. http:// dx.doi.org/10.4025/cienccuidsaude.v\%vi\%i.25445. 
21. Pazin-Filho A, Frezza G, Mstsuno AK, Alcântara ST, Cassiolato S, Bitar JPS, et al. Princípios de prescrição médica hospitalar para estudantes de medicina. Medicina (Ribeirão Preto) [Internet]. 2013 [acesso em: 10 jul. 2018];46(1):183-94. Disponível em: http:// www.revistas.usp.br/rmrp/article/view/62319/65125. https://doi.org/10.11606/issn.2176-7262. v46i1p183-194.

22. Shahrokhi A, Ebrahimpour F, Ghodousi A. Factors effective on medication errors: a nursing view. J Res Pharm Pract [Internet]. 2013 [acesso em: 10 jul. 2018];2(1):18-23. Disponível em: https://www.ncbi. nlm.nih.gov/pmc/articles/PMC4076895/. https://doi. org/10.4103/2279-042X.114084.

23. Brasil. Ministério da Saúde (BR) [Internet]. Brasília (BR). Protocolos básicos de segurança do paciente: prevenção de quedas, identificação do paciente e segurança na prescrição, uso e administraçáo de medicamentos; 2013 [acesso em: 10 jun. 2018]. Disponível em: http:// portalarquivos.saude.gov.br/images/pdf/2014/julho/03/ Protocolo-Medicamentos.pdf.

24. Agência Nacional de Vigilância Sanitária (BR). Assistência segura: uma reflexão teórica aplicada à prática. Brasília (DF); 2013 [acesso em: 12 jun. 2018]. Disponível em: http://portal.anvisa.gov. br/documents/33852/3507912/Caderno $\pm 1 \pm-$ \pm Assistencia \pm Segura \pm- \pm Uma \pm Reflexao \pm Teorica \pm Aplicada $\pm a \pm$ Pratica/97881798-cea0-4974-9d9b077528 ea1573.

25. Leufer T, Cleary-Holdforth J. Let's do no harm: medication errors in nursing: Part 1. Nurse Educ Pract [Internet]. 2013 [acesso em: 11 jul. 2018];13(3):213-16. Disponível em: https://www.ncbi.nlm.nih.gov/pubmed/23474430. https://doi.org/10.1016/j.nepr.2013.01.013. 